Estrategias y recursos empleados por el movimiento estudiantil en el 2011

Violeta Montero Barriga - Carlos Muńoz Labrańa - María Inés Picazo Verdejo

Pp. 137 a 157

\title{
ESTRATEGIAS Y RECURSOS EMPLEADOS POR EL MOVIMIENTO ESTUDIANTIL EN EL $2011^{1}$
}

Strategies and resources employed by the students' movement during 2011

\author{
Violeta Montero Barriga* \\ Carlos Muñoz Labraña** \\ María Inés Picazo Verdejo***
}

\begin{abstract}
RESUMEN
La investigación describe las estrategias y recursos empleados por el movimiento estudiantil chileno en las protestas masivas del año 2011, considerando los aportes y límites de la teoría de movilización de recursos como clave explicativa. En base a la identificación de los repertorios de acción movilizados por los estudiantes chilenos, se propone una caracterización de estrategias internas y externas al movimiento que, en conjunto, den cuenta de una acción política determinada por elementos racionales, al mismo que emotivos y espontáneos. La investigación es eminentemente descriptiva y cualitativa, pues se basa en el análisis de registros de prensa y en focus group efectuados a exdirigentes estudiantiles del 2011.

Palabras clave: estrategias, recursos, movilización estudiantil.

\footnotetext{
${ }^{1}$ Esta investigación se enmarca dentro del Proyecto Fondecyt No 1131132, titulado: "Acción colectiva y políticas públicas en perspectiva: lecciones a partir del movimiento educacional chileno". Los autores agradecen a Luis Fuentes Navarrete, Magíster en Política y Gobierno de la Universidad de Concepción por el trabajo realizado dentro de este proyecto.

* Departamento de Administración Pública y Ciencia Política, Universidad de Concepción. Concepción, Chile. Correo electrónico: violetamontero@udec.cl

** Departamento de Ciencias de la Educación, Universidad de Concepción. Concepción, Chile. Correo electrónico: carlosem@udec.cl

*** Departamento de Administración Pública y Ciencia Política, Universidad de Concepción. Concepción, Chile. Correo electrónico: mpicazo@udec.cl
}

Artículo recibido el 29 de mayo de 2015. Aceptado el 20 de diciembre de 2016. 


\begin{abstract}
The research describes the strategies and resources used by the Chilean student movement in the mass protests of 2011, considering the contributions and limits of resource mobilization theory as an explanatory key. Based on the identification of action repertoires mobilized by the Chilean students, it proposes a characterization of strategies both internal and external to the movement, which altogether account for a certain political action determined by rational elements, as well as emotional and spontaneous ones. Research is basically descriptive and qualitative, since it is based on the analysis of press records and focus group made to former student leaders of 2011.
\end{abstract}

Keywords: Strategies, resources, student mobilization.

\title{
ANTECEDENTES GENERALES Y PROBLEMATIZACIÓN
}

Las movilizaciones universitarias en Chile tienen una trayectoria reivindicativa de antigua data. Ya en la década de los 60 y 70 pero sobre todo durante los ańos 80 en contra de la dictadura, los estudiantes universitarios jugaron un importante rol movilizador.

Con posterioridad al retorno a la democracia, si bien se registraron algunas expresiones de descontento estudiantil como el Primer Reclamo (1997), El Mochilazo (2001) y la Revolución Pingüina (2006), lo ocurrido durante el año 2011, centro de nuestro interés, desborda con creces las movilizaciones efectuadas hasta ese momento por su capacidad de convocatoria estudiantil y ciudadana.

La movilización del 2011 se inicia entre los meses de abril y mayo con una paralización en la Universidad Central, oportunidad en la cual la Confederación de Estudiantes de Chile (CONFECH) estimuló una discusión sobre los problemas que experimentaba el sistema universitario heredado de la dictadura. La crítica efectuada dio origen a un petitorio en el que los estudiantes solicitaban una mayor intervención del Estado en materia educativa, fin al lucro, aumento del gasto público en educación, reforma al sistema de acceso para garantizar igualdad de oportunidades mediante la implementación de mecanismos de ingreso complementarios a la Prueba de Selección Universitaria (PSU), y democratización de las instituciones universitarias a través de la derogación de algunos de los artículos del Decreto de Fuerza Ley No 2 de 2010.

Lo interesante de este período es que la movilización se desarrolló gradualmente y a las pocas semanas, decenas de miles de personas se convocaban solo en la Región Metropolitana (Durán, 2012: 47). En este contexto se produjeron centenares de marchas, paralizaciones de actividades, diversas tomas de establecimientos y múltiples y creativas expresiones de protesta de jóvenes 
universitarios, secundarios y otros tantos actores sociales y políticos, que se sumaron intermitentemente a las acciones colectivas durante al menos siete meses de movilización (UNICEF, 2014).

A modo de ejemplo, en la primera marcha nacional convocada por la CONFECH en Santiago (12 de mayo) participaron unas 15.000 personas. El 1 de junio la columna de manifestantes fue calculada en 20.000 , mientras que a mediados del mismo mes ya había entre 80.000 y 100.000 personas manifestándose en las calles de la capital.

A inicios de junio había tres universidades movilizadas, mientras que a mediados del mismo mes habían alrededor de 17; algunas incluso en paro indefinido o toma de sus dependencias. Hasta octubre de 2011 el movimiento estudiantil había sido capaz de realizar más de 250 eventos de protesta en las principales ciudades del país, movilizando a miles de personas y a un centenar de organizaciones sociales de los más diversos ámbitos del quehacer. En este proceso la capacidad de convocatoria de la CONFECH fue vital, porque logró en forma paulatina mayor apoyo de la ciudadanía y la adhesión de organizaciones sociales con importante presencia a nivel nacional y/o regional como el colegio de Profesores, la Central Unitaria de Trabajadores de Chile (CUT), la Confederación de Trabajadores del Cobre, la Agrupación Nacional de Empleados Fiscales (ANEF) y algunas $\mathrm{ONG}$ 'S, entre otras.

Algunos autores destacan que en este proceso la activación de distintas redes fue fundamental y la consecuencia fue la ampliación del apoyo social, el fortalecimiento de las orgánicas de bases y la diversificación de las estrategias utilizadas.

La investigación propuesta tiene como objetivo precisamente estudiar el movimiento estudiantil a partir del análisis de estos eventos. Particularmente identificar y analizar las estrategias y recursos movilizados en los intensos meses del 2011. Se busca realizar una descripción de las prácticas organizativas desplegadas por los jóvenes movilizados, aquellas que a nuestro juicio, favorecieron la mantención de la movilización y permitieron, entre otros factores, situar las demandas estudiantiles en la agenda pública y obtener el apoyo masivo de los estudiantes y de la ciudadanía. Ahora bien, estas prácticas solo se explican a la luz de la relación establecida entre el movimiento estudiantil y las autoridades políticas. Vale decir, asumimos que estas acciones estudiantiles se constituyen y determinan por la relación de los jóvenes universitarios con representantes de gobierno. En particular, con el gobierno de centro derecha de Sebastián Piñera, quien presentó una determinada concepción de la educación, y que fue duramente cuestionada por los jóvenes movilizados.

En torno a estos eventos de movilización del 2011 hay bastante investigación nacional disponible. Lo novedoso de la presente aproximación 
es que se realiza sometiendo a evaluación la teoría de movilización de recursos (Mc Carthy y Zald 1977, McCarthy, Mayer y Zald, 2001), sus aportes y límites explicativos para entender la movilización estudiantil chilena, sus particularidades y dinámicas de organización y relación. Esta opción investigativa implica revisar el rendimiento analítico de la teoría y abrir la mirada a enfoques latinoamericanos que complementan, o explican de mejor forma la particularidad de esta acción colectiva juvenil.

Consecuentemente, las preguntas que nos orientan son ¿Cuáles fueron las estrategias y recursos utilizados por el movimiento estudiantil en Chile para obtener adhesión y apoyo de estudiantes y ciudadanos durante el 2011? y ¿Cuáles son los aportes y límites de la perspectiva de movilización de recursos para entender este proceso organizativo?

\section{EL MOVIMIENTO ESTUDIANTIL CHILENO Y LA MOVILIZACIÓN DE RECURSOS}

La teoría de la movilización de recursos irrumpió en la escena norteamericana de los años 70 con los trabajos pioneros de McCarthy y Zald (1977), Obsershall (1972) y Tilly (1978); cuestionando enfoques funcionalistas (Parsons, 1968) y de comportamiento colectivo.

La aparición de esta teoría implicó un quiebre con la primacía psicológica que enfatizaba en la frustración, privación, agravio y agresión (Mc Carthy y Zald, 1977; Puricelli, 2005) como factor explicativo clave de la movilización social. Con mayor cercanía a la sociología y economía, esta propuesta observó la movilización del colectivo estudiando con mayor interés las dinámicas y tácticas objetivas del grupo movilizado, que explicaban el crecimiento y declive del mismo. En este contexto, la atención a los recursos y estrategias de los grupos se tornaría central porque permitía entender de mejor forma la dinámica y desarrollo del movimiento.

Desde su aparición, la Teoría de Movilización de Recursos, fue ganando adeptos y provocando la aparición de múltiples trabajos académicos que se concentraron principalmente en el contexto norteamericano, y luego en menor medida, en América Latina.

Siguiendo el análisis de McCarthy y Zald (1977, 2002), la teoría permitiría analizar los factores que influyen en la dinámica de movilización, y que son fundamentalmente tres. Primero, el apoyo social de parte de los adherentes que suscriben los fines de la movilización y de los simpatizantes que aportan a la movilización con recursos de diversa naturaleza. Segundo, los recursos diversos susceptibles de ser utilizados en los discursos y estrategias de movilización, y que pueden ser organizacionales y de acceso a los medios de comunicación. Y tercero, el 
compromiso o alianzas con organizaciones e individuos exteriores al movimiento, como las autoridades públicas o los medios de comunicación, cuya implicación puede promover o debilitar la acción colectiva.

Sobre los aportes de la teoría, podemos decir, siguiendo a Puricelli (2005:3), que permitiría comprender que:

a) Los agravios no necesariamente producen movimientos automáticamente, el proceso es más complicado; b) Los integrantes no son irracionales, psicológicamente padecidos, sino participantes informados y convencidos; c) Los movimientos no son entidades aisladas, sino que interactúan con otras organizaciones, autoridades, partidos, sectores de la sociedad, los medios de comunicación, etcétera, y usan a la infraestructura de la sociedad para movilizarse.

A nuestro juicio, el aporte de la teoría es que complejiza el análisis respecto de los movimientos sociales, abandonando la mirada unicausal que los asume como expresión psicológica del malestar. Al contrario, desde esta perspectiva los movimientos sociales serían también una opción política, razonada y evaluada por sus integrantes como un acto necesario y útil en pro de conseguir ciertos objetivos. El desafío sería identificar y analizar estos componentes internos del movimiento y describirlos considerando su importancia en el cumplimiento de los fines colectivos propuestos.

Las principales críticas son que esta teoría deja de lado la ideología, pues se concentra en la identificación de elementos objetivos y racionales. Bajo este prisma, la existencia de factores subjetivos de los movimientos no tendrían cabida, pues muy en sintonía con la teoría de la elección racional de Mancur Olson (1965), se estudiaría un sujeto que saca cálculos de costo y beneficio para determinar sus acciones, sin considerar actuaciones bajo principios filantrópicos o altruistas.

Es justamente este reduccionismo el que critican autores como Puricelli (2005) Svampa (2009), Zibechi (2012), Valenzuela (2012), entre otros, refiriéndose a la particularidad de los movimientos sociales en América Latina, los que motivados inicialmente por situaciones de agravio o sentimiento de injusticia, se constituirían gradualmente en actores políticos relevantes, sin contar necesariamente con tantos recursos materiales e inspirados por discursos globales de cambio social.

Para el análisis del movimiento estudiantil en Chile 2011 asumimos esas críticas, proponiendo una mirada teórica complementaria, basada en los siguientes postulados. En primer lugar, en sintonía con el modelo de análisis del proceso político, asumimos que los movimientos sociales se desarrollan siempre en interacción con una estructura política que abre o cierra oportunidades a los grupos movilizados. El movimiento social implicaría entonces desafiar o interpelar a determinados actores del sistema político y/o a los consensos respecto del orden establecido. Por tanto, la definición de estrategias y utilización de recursos estaría determinada por la lectura 
que los actores movilizados hacen de la estructura en medio de la contienda política. La propuesta es entonces observar lo micro, la capacidad de agencia de los actores movilizados, pero siempre en relación con lo macro. Es decir, se estudiarán las estrategias y recursos movilizados por los estudiantes en Chile durante el año 2011, en interacción con el gobierno de centro derecha de Sebastián Piñera.

En segundo lugar, entendemos que los movimientos sociales se constituyen para enfrentar estructuras de poder y un sistema político que no es receptivo a sus demandas. La movilización sería entonces una acción política, un esfuerzo deliberado por alcanzar objetivos propuestos $y$, por tanto, no debe ser entendida como una conducta desviada, tal como postulaban enfoques sobre movimientos sociales, propios de los años 60 en Norteamérica. La movilización es fruto del interés de intervenir en la política, incidiendo en la gestión y decisión de un conflicto; por tanto, representa un esfuerzo de incidencia en la sociedad civil y en el sistema político.

En tercer lugar, compartimos con la Teoría de Movilización de Recursos la relevancia de describir la acción de movimientos sociales a través de las estrategias y recursos movilizados; sin embargo, consideramos que esta perspectiva no es suficiente para entender lo sucedido en el 2011 y debe ser complementada. Tal como señala Jasper (2012) esto es así porque el valor explicativo de las grandes teorías sobre movimientos sociales está siendo reexaminado y es necesario dar espacio a otras aproximaciones culturalistas que reconocen y dotan de valor aspectos, por ejemplo, como las emociones.

Asumir un trabajo bajo estos postulados implica estudiar aspectos internos a los grupos movilizados, incluyendo variables tales como organización, intereses, recursos, oportunidades y estrategias. Implica preguntarnos principalmente sobre los recursos propios de los estudiantes y aquellos obtenidos en interacción con otros para sustentar la movilización en el tiempo; y sobre aquellas estrategias elaboradas colectivamente y/o de manera más autónoma o espontánea por intergrantes del colectivo. Para mayor precisión conviene profundizar claramente señalando qué entendemos por recursos y estrategias.

Por estrategias, siguiendo a Cefaï (2011) entenderemos aquellas acciones y procedimientos que maximizan la relación medios-fines, y que buscan de manera planificada, instalar la problemática educativa en el conjunto de los estudiantes y ciudadanos, superando la dispersión, con el fin de concretar intereses. Así, tal como indica el trabajo clásico de Mc Carthy y Zald (1977), las estrategias incluyen la movilización de simpatizantes y la neutralización y/o transformación de la masa y los públicos de elite en simpatizantes. En el proceso, complejo por su dinámica interna, ocurren múltiples dilemas; por ejemplo la elección de tácticas en tanto alguna de estas puede entrar en contradicción con objetivos particulares. Y el establecimiento de relaciones intra y extra organización, en tanto se condicionan por intereses, cooperación o competencia. A nuestro juicio, las estrategias pueden ser entendidas ampliamente bajo la noción de "repertorios de acción colectiva". Esto es posible dada 
la productividad analítica de esta noción que señala la importancia de considerar aspectos o dimensiones estructurales, culturales y simbólicas. Los repertorios de acción, configuran un horizonte de experiencia colectiva que se aprende en la práctica, en la protesta, en las marchas públicas, en las reuniones colectivas, vale decir, en la movilización. En el caso del movimiento estudiantil del 2011 en Chile, las prácticas de los jóvenes ya tenían antecedentes, pues el año 2006, en el contexto de la llamada Revolución Pingüina se llevaron a cabo una serie de acciones colectivas transgresivas, además de interacciones con la autoridad política.

Por su parte, el concepto de recursos hace alusión a los medios con que cuenta la organización para llevar a cabo sus estrategias. Por tanto, su consideración siempre debe darse en relación a los fines y tácticas propuestas. Según Golsorkhi y otros (2011) los recursos son definidos como el conjunto de factores/variables que son utilizados por el movimiento, que pueden controlar o no, y que explican las acciones que los actores emprenden para controlarlos en pro de sus objetivos. En la movilización social el principal recurso considerado son los propios miembros de la organización comprometidos; sin embargo, también apreciamos el despliegue de recursos materiales, pertenecientes a los propios jóvenes movilizados y/o a redes externas. Y recursos simbólicos, entre los que se cuentan la solidaridad, la cohesión emocional, la disciplina y el compromiso de los miembros que van desarrollándose a través del lenguaje y la comunicación grupal.

Con todo, postulamos que es posible e interesante estudiar las estrategias y recursos movilizados por los estudiantes chilenos en el contexto del año 2011, pues valorando este esfuerzo descriptivo y de caracterización aportamos a la visibilización de componentes micro, que en conjunto permitirían explicar la efectividad de esta movilización que duró casi ocho meses de protesta ininterrumpida y que impactó distintas esferas de la sociedad e institucionalidad chilena.

\section{METODOLOGÍA}

Los datos que dieron vida a la investigación fueron recogidos durante el bienio 2014-2015 y se desarrolla bajo un paradigma cualitativo e interpretativo. Analiza la experiencia de movilización estudiantil del año 2011, desde la perspectiva de los propios jóvenes movilizados, recurriendo a sus declaraciones en la prensa tanto escrita como digital, tanto oficialista como de oposición. También incluye el análisis de aquellos documentos (declaraciones) emanados de sus propias organizaciones de base y la nutrida información hecha circular - por los propios estudiantes- a través de distintas páginas web y redes sociales. A lo que habría que agregar el análisis de las publicaciones escritas en primera persona por los propios líderes del movimiento (Jackson, 2013; Figueroa, 2013), así como la realización de cinco focus group. 
El trabajo documental en la prensa, páginas web y redes sociales, como de la bibliografía asociada, se realizó mayoritariamente en Concepción. Mientras que los focus group fueron efectuados en cinco ciudades del país: Valparaíso, Santiago, Talca, Concepción y Puerto Montt.

Los criterios empleados para la selección de la base documental fueron el carácter primario de la fuente, la procedencia de la información y su posibilidad de triangulación, así como su contemporaneidad con el proceso en estudio. Los criterios empleados para la selección de la muestra de las personas que participaron en los focus group fueron: a) Haber participado activamente de las acciones colectivas del movimiento en el ańo 2011; b) Haber participado en cualquiera de las siguientes categorías: dirigente, estudiante o simpatizante; c) Ser mayor de edad; y, d) Tener disponibilidad para reconstruir el recuerdo de la acción.

Los documentos fueron procesados a través del análisis de contenido, mientras que para el análisis de los focus group, luego de ser grabados y transcritos, se utilizó la técnica ad-hoc (Kvale, 2007: 184-185), que consiste en la combinación de diversas formas de análisis según las características de cada uno: interpretación, categorización u otras técnicas tales como la concentración de significados o la construcción de tipos ideales.

Las categorías de análisis fueron establecidas en su mayoría de manera emergente, a partir de la revisión de las fuentes disponibles; sin embargo, también hay una propuesta original de caracterización de las estrategias, basada en consideraciones organizativas y económicas (Cardona, 2011). Estas categorías de análisis orientan la presentación de los resultados a través de un ejercicio descriptivo y analítico que se expone a continuación. Las categorías son las siguientes:

\begin{tabular}{|l|l|l|}
\hline Estrategias & Internas & Externas \\
\hline De penetración & $\begin{array}{l}\text { 1. Ampliar el movimiento más allá } \\
\text { de la CONFECH. }\end{array}$ & $\begin{array}{l}\text { 1. Expandir el movimiento más allá } \\
\text { del mundo estudiantil. }\end{array}$ \\
\hline De comunicación & $\begin{array}{l}\text { 2. Generar mecanismos alternativos } \\
\text { de información. }\end{array}$ & $\begin{array}{l}\text { 2. Marcar presencia en los medios } \\
\text { de comunicación tradicional. }\end{array}$ \\
\hline De relación & $\begin{array}{l}\text { 3. Expandir territorialmente el } \\
\text { movimiento. }\end{array}$ & $\begin{array}{l}\text { 3. Promover nuevas prácticas } \\
\text { políticas. }\end{array}$ \\
\hline De integración & $\begin{array}{l}\text { 4. Evitar la personalización del } \\
\text { movimiento. }\end{array}$ & $\begin{array}{l}\text { 4. Utilizar un discurso inclusivo y } \\
\text { comprensible por la ciudadanía. }\end{array}$ \\
\hline De atención & & $\begin{array}{l}\text { 5. Responder a los emplazamientos } \\
\text { e intentos de desmovilización del } \\
\text { Gobierno. }\end{array}$ \\
\hline
\end{tabular}




\section{ANÁLISIS DE RESULTADOS}

Como se mencionó con anterioridad, proponemos el análisis de estrategias y recursos internos y externos al movimiento (los primeros dirigidos al mundo estudiantil y los segundos a la ciudadanía), categorizados de acuerdo a los fines de la respectiva estrategia. Se consideran estrategias de penetración, destinadas a expandir el movimiento tanto hacia los propios jóvenes universitarios como más allá de él; de comunicación encaminadas a asegurar el flujo expedito de la información; de relación orientadas a vincular territorial e ideológicamente el movimiento a través de nuevas prácticas políticas; de integración destinadas a generar un movimiento inclusivo que fuera más allá de las propias organizaciones juveniles y de atención encaminadas a responder al gobierno.

\section{Estrategias internas. Dirigidas a obtener el apoyo del mundo estudiantil}

Ampliar el movimiento más allá de la CONFECH. Esta estrategia pretendía ampliar la base de apoyo del movimiento estudiantil entre los miembros del grupo de referencia (Olson, 1965), los estudiantes, los endeudados; más allá de las diferencias entre universidades públicas o privadas. Además buscaba solidaridad interpelando a potenciales simpatizantes y/o redes de apoyo entre otros actores del sistema de educación superior y entre estudiantes secundarios. Esta estrategia es necesaria tal como sugiere el análisis clásico de Mc Carthy y Zald (1977), pues permite desarrollar el movimiento y se lleva a cabo a través del uso de diversos recursos organizacionales y materiales. Veamos algunos ejemplos.

Entre los estudiantes universitarios movilizados existía la convicción de que era necesario ampliar la base de apoyo del movimiento y que el desafío era romper la distinción entre jóvenes pertenecientes a universidades tradicionales versus privadas. Esta separación se expresaba en la Confederación de Estudiantes de Chile (CONFECH), principal organización emprendedora del movimiento, pues desde su creación en 1984, se incluían solo estudiantes de universidades chilenas organizados en federaciones democráticamente elegidas, dejando fuera a quienes estudiaban en los Centros de Formación Técnica (CFT), Institutos Profesionales (IP), Universidades Privadas y estudiantes secundarios. En el año 2011, como mencionamos, la estrategia de la CONFECH fue contactar y coordinar reuniones con estos estudiantes con el propósito de convocarlos a plegarse a la movilización. Fue una tarea compleja de realizar, dada la histórica distancia y los prejuicios existentes entre los propios dirigentes universitarios, como lo expresa la siguiente afirmación:

fue un tema complejo ... todos sabíamos que teníamos que ampliar la base del movimiento pero había distintas opiniones al respecto, algunos pensaban que 
los estudiantes de dichos planteles no tenían la madurez política ni las mínimas condiciones para participar. De hecho algunos centros de alumnos de esos planteles eran designados o muy cercanos a las autoridades, otros firmaban al matricularse una cláusula en donde se comprometían a no participar de las marchas, y otros ni siquiera tenían organización estudiantil porque no les interesaba (Dirigente estudiantil de Concepción, entrevista junio 2014).

Esta acción, a pesar de que significó una gran inversión de tiempo a los universitarios federados, se resolvió al margen del movimiento, pues fueron los propios estudiantes de universidades privadas, centros de formación técnica e institutos profesionales, los que solicitaron plegarse al movimiento, y más aún, en algunos casos se sumaron a la paralización de actividades y otras acciones colectivas ocasionales.

La vinculación con los secundarios fue más fácil de concretar, especialmente por su alto nivel organizativo, la experiencia política de sus líderes y también, porque durante el Movimiento Pingüino del 2006, varias organizaciones secundarias habían generado contactos y apoyado las reivindicaciones estudiantiles que sirvieron de base al movimiento. La estrategia se orientó a tomar contacto con los líderes de las coordinadoras secundarias en forma directa, y de acuerdo a las afinidades políticas de cada uno. Algunos fueron a la CONES mientras que otros, entre los cuales estaban los autodenominados "los sin fechs" grupos organizados en las universidades al margen de sus respectivas federaciones de estudiantes, fueron a la ACES. Como resultado de esta estrategia se incorporaron al movimiento los estudiantes secundarios (municipalizados, particulares subvencionados e incluso algunos particulares pagados) congregados en torno a la CONES y a la ACES, los que entre junio y julio se habían tomado más de 600 colegios en todo el país (EMOL, 2011).

Respecto del vínculo con otros actores del sistema educativo, la CONFECH recibió apoyo de algunos académicos, funcionarios y directivos, incluso simpatía de algunos rectores de universidades públicas. También estableció vínculos con otras organizaciones sociales preocupadas por el derecho a la educación como el Movimiento de Estudiantes de Educación Superior Privada (MESUP) y el Colectivo por la Acción para la Educación de las Personas Sordas (CAEDSOR).

Generar mecanismos alternativos de información. Desde la creación de la CONFECH, una de las críticas que se hizo notar entre las federaciones participantes fue la alta concentración de los medios de comunicación y la carencia de medios informativos alternativos. Con el uso masificado de los aparatos celulares, el desarrollo de las TICs y las redes sociales, el problema de la falta de acceso y circulación de la información tendió a modificarse, como quedó demostrado 
en el Movimiento Pingüino del 2006 (Valderrama, 2013). El 2011 los jóvenes movilizados aprovecharon su experiencia teconológica diseñando estrategias técnicas que permitieron llegar con la información en forma rápida y expedita, a través del uso intensivo de recursos materiales asociados a la telefonía móvil, internet y redes sociales. El uso de mensajería instantánea, de correos electrónicos y de redes sociales como twitter, facebook, instagram, fotologs, fue habitual entre los jóvenes movilizados y sirvió, como en otras experiencias de protesta social, en la coordinación, convocatoria y organización de las acciones colectivas (Tilly, 2009).

En la época se crearon distintas plataformas como "movilizatechile", "adelantechile" y "yodebo" que entregaban información y promovían organización entre estudiantes. A la fecha, además, se mantienen las páginas www.plebiscitoporlaeduacion.cl y https://confech.wordpress.com/noticias/ que muestran distintas visiones y estrategias de estudiantes y su vínculo con otros actores sociales.

Si bien no es nuestro objetivo medir el impacto de las redes sociales en la política (Torres Nabel, 2015), es posible señalar que el uso de teconologías de información generó una suerte de ciberactivismo favoreciendo la coordinación, articulación y difusión de las actividades dentro del mundo estudiantil, sus adherentes y simpatizantes, superando las formas tradicionales de comunicación.

Expandir territorialmente el movimiento. La dinámica que fue adquiriendo el movimiento exigía difundir el diagnóstico, y las demandas sobre la realidad educacional, a todo el país. El convencimiento fue que "el problema de la centralización también afectaba al movimiento" (Dirigente estudiantil Puerto Montt, 2011. Disponible en archivo Chile "El movimiento estudiantil más allá de la Confech") y la estrategia fue en cierta medida reactiva a las tensiones por exigencias de participación e inclusión de distintos actores estudiantiles, principalmente de universidades regionales.

Para enfrentar lo anterior, se toma contacto con líderes estudiantiles regionales pertenecientes a organizaciones adscritas a la CONFECH; con jóvenes miembros de partidos políticos de la oposición (PS, PC, DC, MAS y el PR); y con otras organizaciones presentes en las principales universidades adscritas al Consejo de Rectores de las Universidades Chilenas (CRUCH) como la Fuerza Universitaria Rebelde (FUR), el Frente de Estudiantes Libertarios (FEL), el Movimiento Universitario de Izquierda (MUI), entre otras.

Así, el movimiento se extendió a universidades ubicadas tanto en el norte como en el centro y sur del país, alcanzado a ciudades como Arica, Iquique, Copiapó, La Serena, Valparaíso, Talca, Chillán, Concepción, Temuco, Osorno y Punta Arenas lo que implicó la realización de asambleas de la CONFECH en estas ciudades. 
Evitar la personalización del movimiento. La crítica a las formas tradicionales de ejercicio político cupular impactaron en la organización de los jóvenes movilizados. La estrategia implicó un desafío en términos simbólicos y organizativos y, es a nuestro juicio el aspecto donde radica la particularidad central del movimiento estudiantil chileno, que no puede explicarse a través de la lógica racional y meramente cognitiva.

Tal como plantea Zibechi $(2006,2012)$ la subjetividad política de los movimientos latinoamericanos, implican el ejercicio de nuevas prácticas, más horizontales, autónomas, de participación colectiva y de ejercicio de democracia directa, en oposición a estructuras de organización jerárquicas y tradicionales.

Efectivamente, en el movimiento estudiantil del 2011 la idea fue ir más allá de los rostros y reconocidos liderazgos que tempranamente se dejaron sentir en los medios de comunicación como el de Camila Vallejos -escogida el personaje del año por The Guardian- o de Giorgio Jackson presidente de la FEUC, y privilegiar la figura de la asamblea ampliada y las vocerías como formas más democráticas y participativas de democracia interna. La asamblea fue un espacio de discusión y órgano resolutivo; y las vocerías la modalidad para dar a conocer las resoluciones a los estudiantes y a la ciudadanía.

El resultado fue un movimiento con dinámicas mas lentas en términos de negociación política, pero que a nivel interno, si bien estaba integrado por estudiantes con ideales y distintas visiones políticas - muchas veces en contraposición y conflicto- logró implementar una vocación democrática y amplia participación de las bases (Valenzuela, 2012).

\section{Estrategias externas. Dirigidas a obtener el apoyo de la ciudadanía}

Expandir el movimiento más allá del mundo estudiantil. Una de las principales características del año 2011 es que se estableció un movimiento con vínculos más allá de los estudiantes. Según señala Camila Vallejo "el movimiento que surgió en el 2011 en Chile no fue un movimiento espontáneo ni sólo de jóvenes, sino una síntesis de una larga lucha de estudiantes y trabajadores de nuestro país" (Intervención en Foro Internacional Emancipación e Igualdad, Buenos Aires, 2015) que planteaban la necesidad de recuperar y fortalecer la educación pública.

Los líderes del movimiento 2011 advierten que existe un sentimiento de injusticia y malestar (Mayol, 2012) que puede ser aprovechado para sumar adherentes y ampliar la base del movimiento. De allí el despliegue de representantes del movimiento estudiantil en asambleas de organizaciones sociales participando en encuentros, foros, paneles y debates, así como en marchas en apoyo a sus demandas sectoriales, por ejemplo, la participación de estudiantes en marcha realizada por la Confederación Nacional de Funcionarios de Salud Municipalizada CONFUSAM, en demanda de mejoras salariales (13 de septiembre). 
Por su parte, a través de sus propias redes sociales y páginas web, el movimiento estudiantil alimentó la idea de un movimiento social amplio e inclusivo que estaba dispuesto a enfrentar las estructuras de poder que no estaban siendo receptivas a sus demandas.

Como producto de esta estrategia se fueron plegando al movimiento diversas organizaciones como el Colegio de Profesores, los trabajadores en huelga de la principal productora de cobre en el país CODELCO, la Central Unitaria de Trabajadores de Chile (CUT), la Agrupación Nacional de Empleados Fiscales (ANEF), entre otras.

El momento de mayor adhesión social fue con motivo del llamado a paro convocado por la CONFECH el 9 de agosto, donde se sumó el Colegio de Profesores, la CUT y la ANEF, y otras 42 organizaciones entre las que se cuentan Acción Ecológica, Agrupación de Familiares de Ejecutados Políticos, Asociación Nacional de Defensa de los Derechos de los Consumidores y Usuarios de la Seguridad Social, Asociación Nacional de Académicos e Intelectuales de Chile, Asociación Chilena de Barrios y Zonas Patrimoniales, el Centro de Estudios para el Desarrollo de la Mujer, el Colegio de Periodistas y la Confederación Nacional de Funcionarios de Salud Municipalizada (Le Monde diplomatique, 2011).

Tener presencia en los medios de comunicación tradicional. Aunque el movimiento procuró que la implementación de los medios alternativos de comunicación dirigidos al estudiantado estuvieran abiertos a la ciudadanía, jamás subestimó la importancia que poseían los medios de comunicación tradicional como la prensa escrita, radial, televisiva y digital. De allí que desde un comienzo hubo una especial preocupación por tener presencia permanente en los medios, mediante la realización de puntos de prensa realizados en lugares, días y horas difundidas profusamente; la asignación de voceros encargados de dar cuenta de los acuerdos logrados a los distintos medios luego de cada asamblea plenaria; y el envío de representantes con mandatos específicos a programas radiales y televisivos. En este último contexto existen muchos registros de la participación de los representantes estudiantiles en programas televisivos como la participación del vicepresidente de la FECH, Francisco Figueroa, en un programa transmitido por CNN Chile, el día 22 de agosto de 2011, que provocó la ira del exministro de Educación del Presidente Ricardo Lagos en el momento que le recordó que él había firmado el cuestionado sistema de Crédito con Aval del Estado (CAE) que tenía endeudado a los estudiantes y sus familias.

La utilización de los medios tradicionales de información se realizó para dar cuenta del pensamiento y actividades realizadas por el movimiento; reaccionar y responder a las propuestas y dichos realizados por el gobierno de Pińera; y responder a otros medios u actores proclives al gobierno. A modo de ejemplo, a solo días de que 
el Ministro de Educación presentara un documento con propuestas para solucionar las peticiones de los alumnos, la CONFECH junto a los secundarios y el Consejo de Rectores resolvió rechazar la propuesta con amplia cobertura comunicacional. Por su parte, destacan múltiples reacciones mediáticas que tuvieron los estudiantes ante informaciones o editoriales de prensa que atacaban al movimiento, siendo el diario El Mercurio, EMOL uno de los más fecundos (Pérez, 2013). Por ejemplo en agosto de 2011 luego de que EMOL publicara una noticia bajo el título "Movilizaciones estudiantiles: todo programado desde marzo", donde se afirma que las movilizaciones estudiantiles obedecían a un plan maquinado de acción y a un programa previo, la CONFECH respondió evidenciando que la información utilizada por el Mercurio era la agenda de la FECH que resumía el trabajo realizado por la organización estudiantil en el 2010. De este modo se dio cuenta del error en distintos medios informativos defendiéndose así de lo que consideraban una acusación infundada.

Utilizar un discurso inclusivo y comprensible por la ciudadanía. El objetivo fue llegar a la ciudadanía a través de un discurso sencillo, directo y dirigido al individuo, procurando hacer comprensible el diagnóstico, los fines, los objetivos y los medios utilizados por el movimiento.

La idea fue reinterpretar la realidad e instalar nuevos sentidos y significados en torno a ella, especialmente a través de ciertas palabras que en forma de eslogan fueron recurrentemente utilizados en los discursos y declaraciones públicas, en los lienzos confeccionados por estudiantes a lo largo del país y en los numerosos documentos preparados para informar a la opinión pública sobre los fines del movimiento. El propósito fue facilitar el recuerdo, llamar la atención de la comunidad e instalar algunas ideas claves del movimiento, con el propósito de generar un discurso colectivo sobre la situación educativa. En este contexto destacan eslóganes con un alto valor simbólico como "La educación es un derecho y no un privilegio", "una educación que lucra vulnera el estado de derecho" , "educación libre, digna y gratuita"; "la educación no se vende", "la educación no es un bien de consumo", "no somos una minoría, somos una nación", entre otros. Además, ampliando la mirada del movimiento a otros aspectos sociopolíticos, destacan eslogan como "se va a acabar la educación de Pinochet" y "El pueblo unido jamás será vencido" haciendo alusión a la Dictadura en Chile y protestas sociales anteriores.

A nuestro juicio, en la elaboración de los mensajes se juega gran parte de la producción simbólica y emotiva del movimiento (Jasper, 2012), pues un acto creativo y una expresión cultural de los jóvenes participantes. La masificación del mensaje a través del eslogan permitió difundir de manera exitosa y comprensible los propósitos de la movilización, mostrando además una efectiva estrategia de difusión cultural que evidenciaba dominio del tema y también emotividad. 
Superar viejas prácticas políticas y promover otras nuevas. La relación entre movimientos sociales y partidos políticos (Somuano, 2007) se ha estudiado actualmente en su complejidad, debido a la emergencia de los primeros y a la crisis de representación y legitimidad de los segundos. Entre ambos hay vínculos y resistencias en un contexto de interacción permanente que fue evidente en el año 2011, como lo demuestra Avendaño (2014). Por una parte, los estudiantes critican las prácticas tradicionales de ejercicio político cupular, el centralismo y la inclinación a la democracia representativa de los partidos políticos, más que a la participación amplia y ciudadana. Por otra parte, algunos estudiantes apostaban a la intermediación de los partidos políticos con el fin de lograr más efectivamente las reivindicaciones educacionales.

Las tensiones existentes hicieron que el movimiento, si bien nunca se distanció efectivamente de los partidos políticos (en algunos de los cuales militaban sus propios dirigentes), asumiera la estrategia de proponer a la ciudadanía otras nuevas formas de hacer política, intentando que el movimiento fuera leído como un movimiento transversal, social y ciudadano, más que partidista. Varias fueron las acciones encaminadas a lograr este objetivo, entre ellas la negación a que los partidos y sus representantes actuaran como mediadores en el conflicto, los esfuerzos por instalar el movimiento como un movimiento social más que político, el rechazo a realizar asambleas en las sede de los partidos, la condena al aprovechamiento realizado por algunos políticos a propósito de algunas acciones y reacciones generadas durante el conflicto y los permanente llamados a "escuchar a las bases" (agosto 2011, Disponible en archivo Chile "El movimiento estudiantil más allá de la CONFECH”).

En este contexto se explica también los esfuerzos por reforzar la centralidad de los movimientos sociales. Por un lado, teniendo una agenda propia como estudiantes. Y por otro, insertando las demandas sectoriales en otras manifestaciones de movimientos sociales iniciados en forma previa al movimiento y con posterioridad a él, como el movimiento efectuado en Magallanes en contra del alza del gas, en enero de 2011; el movimiento de protesta de los damnificados por el terremoto y maremoto de febrero de 2010; el realizado a favor de quienes protestaban por la aprobación de un megaproyecto en la Patagonia "Hidroaysén", entre otros.

La confluencia de actores sociales de protesta no pasó inadvertida para la opinión pública ni para los académicos por lo que existió un gran interés y sorpresa por estas expresiones llegando algunos a plantear que nos encontrábamos ante "un nuevo despertar de los movimientos sociales en Chile" (Grez, 2011).

Responder a los emplazamientos e intentos de desmovilización del Gobierno. La interacción con la autoridad política es parte inevitable de toda movilización. 
Más aún, en la lógica de desafiadores y contendores, de la "contienda política" (Tilly 2009; McCarthy y Zald, 1977; Tarrow, 1994), se entiende que gran parte del desafío de los movimientos sociales es responder oportuna y estratégicamente a las autoridades políticas con el fin de alcanzar los objetivos propuestos.

Por tanto, además de asumir las dificultades internas propias de cada movimiento, (en este caso de un movimiento donde participaban estudiantes con distintas posiciones, trabajadores y organizaciones sociales diversas), el principal problema era definir la forma en que se debía abordar la relación con el gobierno y con la institucionalidad política.

Una posición al interior del movimiento estudiantil en el 2011 insistía en la premisa del "todo o nada" y de la confrontación con la autoridad por la defensa del petitorio planteado y de los principios del movimiento (Picazo, 2013; Valenzuela, 2012). Por otra parte, otros actores abrían a la posibilidad de mayor diálogo y de negociación. Esta pugna de intereses y posiciones explica la dificultad de resolver este conflicto rápidamente (que se mantiene en distintos aspectos hasta la actualidad) y la duración de la movilización estudiantil en el año 2011.

Sin desconocer la relevancia explicativa de este análisis, en un ejercicio descriptivo, podemos señalar que en el 2011 el movimiento estudiantil mantuvo una actitud de alerta frente a las intervenciones realizadas por los personeros de gobierno, algunas de las cuales tenían como objetivo central desacreditar directamente la legitimidad, fines y procedimientos del grupo movilizado. En el 2011 no fue posible negociar acuerdos, pues el peso de las posiciones ideológicas, las distintas concepciones sobre educación y la oposición entre distintas formas organizacionales y relacionales no lo permitían. Muchas veces, por tanto, se dio un diálogo de sordos, y se elaboraron múltiples propuestas sin ningún destino.

El ejemplo más claro se produjo en la presentación de la propuesta GANE, Gran Acuerdo por la Educación (julio de 2011) que fue inmediatamente descartada por el movimiento, por considerar que no incluía plazos, recursos ni procedimientos, y se presentó una contrapropuesta, bajo el nombre de Bases para un Acuerdo Social por la Educación Chilena (julio de 2011). Esta fue rechazada por el Gobierno, quien presentó una nueva propuesta denominada Políticas y Propuestas de Acción para el Desarrollo de la Educación Chilena, que fue también rechazado por los estudiantes. Luego, el ejecutivo presenta su tercera propuesta denominada Medidas del Gobierno en Educación.

Como puede verse en este pequeño relato, la relación entre estudiantes movilizados y sus detractores fue compleja, no siempre se guió por análisis racionales y argumentos cognitivos; sino que también por reacciones emocionales y ajustes pragmáticos (Cefaï, 2007; Emirbayer y Goodwin, 1994). No hubo por tanto, una estrategia clara de negociación; sino más bien reacciones y utilización de recursos de debilitamiento del adversario. Esto implicó que se quebraran los espacios de 
negociación y diálogo, y que se imposibilitara la búsqueda de soluciones. Así, a pesar que las tomas de establecimientos se dejaron y se retomaron las clases, las expectativas del movimiento estudiantil a largo plazo, fueron asumidas como promesas de campaña y explican en parte el actual gobierno de Michelle Bachelet (2014-2018) y las presiones aún persistentes en el campo educativo.

\section{CONCLUSIONES}

El movimiento estudiantil del año 2011 es un caso interesante que ha sido sometido a diversos análisis en el intento de entender su génesis, sus demandas, sus prácticas, sus impactos, entre otros. Nuestro esfuerzo eminentemente descriptivo, ha buscado comprender los repertorios de acción que los jóvenes movilizados utilizaron y que explican las prácticas internas, y la relación con la ciudadanía y el gobierno, desde la perspectiva teórica de la movilización de recursos.

A nuestro juicio, la teoría de la movilización de recursos poseevalor explicativo en tanto concentra la atención en aquellos aspectos micro organizacionales que despliegan los actores movilizados. Asimismo, pone atención en los recursos que se utilizan y confrontan con el fin de lograr cumplir con los objetivos propuestos. En efecto, en este trabajo, proponemos una lectura de las estrategias desarrolladas por los jóvenes y las analizamos a la luz de los antecedentes recabados sobre el movimiento del 2011.

El movimiento estudiantil utilizó diversas estrategias para captar la atención de distintos públicos objetivos: estudiantes, ciudadanía y gobierno. En el primer caso las estrategias utilizadas tuvieron como objetivo llegar especialmente a aquellos estudiantes que no formaban parte de la CONFECH, pero además a quienes no militaban en los partidos políticos ni eran dirigentes, mediante la generación de mecanismos alternativos y autónomos de información y el cuidado de no personalizar el movimiento en figuras particulares, por muy destacada que haya sido su actuación.

En todas las estrategias utilizadas hacia los estudiantes se procura interpelar en forma directa al individuo, apelando a una racionalidad instrumental, a sus biografías personales y familiares y a la importancia de permanecer unidos y comunicados.

En el segundo caso, en el de la ciudadanía, las estrategias estuvieron dirigidas a expandir el movimiento más allá del mundo estudiantil, no descuidar la presencia en los medios masivos de comunicación, utilizar un discurso inclusivo y finalmente realizar acciones tendientes a superar las prácticas tradicionalmente utilizadas para hacer política. Con este segundo grupo de estrategias se buscó ampliar la base del movimiento generando empatía con las demandas, mediante el uso de expresiones artísticas y lúdicas, con una alta carga simbólica y menos racional. 
En el tercer y último caso, en el de las estrategias empleadas con el propósito de dar respuesta a los emplazamientos e intentos de desmovilización del Gobierno, las estrategias utilizadas fueron más concretas, y basadas en datos empíricos con un alto respaldo científico gracias a la conformación de redes con expertos afines con las ideas de los jóvenes. Sin embargo, también durante varios meses de 2011 se apreció la dificultad de negociar en base a argumentos racionales primando el rechazo a la posición ideológica.

A nuestro juicio, en base a lo estudiado, no es posible entender los hechos solo con la perspectiva de la movilización de recursos y análisis racional. No permite incluir aquellos elementos simbólicos, emotivos y pragmáticos presentes en el accionar de los jóvenes agrupados en la movilización del 2011. Esto, pues más que esfuerzos meramente cognitivos y racionales, el 2011 en Chile observamos la emergencia de un discurso basado en un malestar social que implicaba una fuerte carga emocional. Las decisiones organizativas, se situaban también en esta posición, pues partiendo de la crítica a las formas tradicionales de ejercicio político, inauguraron modalidades más cercanas a la democracia participativa. Siguiendo a Zibechi citado por Valenzuela (2012), podemos decir que el año 2011 en Chile apreciamos la emergencia de nuevas líneas de acción que ya habían estado presentes en otros movimientos sociales latinoamericanos, caracterizados por la aspiración de superar las prácticas políticas tradicionales, mediante el uso de recursos no solo materiales y humanos sino que también simbólicos, instalando una subjetividad política caracterizada por la horizontalidad, autonomía y participación colectiva, entre otros factores.

\section{REFERENCIAS}

Avendańo, Octavio. "Fracturas y representación política en el movimiento estudiantil. Chile 2011”, Revista Ultima Década, 41 (2014): 41-68.

Cardona, Raúl. "Estrategias basada en los recursos y capacidades. Criterios de evaluación y el proceso de desarrollo", Revista Forum Doctoral, 4 (2011): 113-147. [Consultado el 07 de mayo de 2015].

Cefaï, Daniel. Pourquoi se mobilise-t-on? Théories de l'action collective. Paris : La Découverte, 2007.

Cefaï, Daniel. "Diez propuestas para el estudio de las movilizaciones colectivas. De la experiencia al compromiso", Revista de Sociología, 26 (2011): 137-166. 
Durán, Carlos. (2012). "El acontecimiento estudiantil y el viraje del proceso sociopolítico chileno", Publicación OSAL (CLACSO), Año XIII/31 (2012) $39-59$.

Emirbayer, Mustafa \& Goodwin, Jeff. "Network Analysis, Culture, and the problema of Agency". En The American Journal of Sociology, Vol 99, 6 (1994) 1411-1454.

Figueroa, Francisco. Llegamos para quedarnos. Crónicas de la revuelta estudiantil. Santiago de Chile: LOM, 2013.

Golsorskhi, Damon et al. (2011) "Mouvements sociaux, organisations et strategies", Revue francaise de gestion, 217 (2011): 79-91.

Grez, Sergio. "Un nuevo amanecer de los movimientos sociales en Chile". Disponible en http://www.uchile.cl/noticias/74720/sergio-grez-toso-unnuevo-amanecer-en-los-movimientos-sociales, 2011. [Recuperado el 23 de marzo de 2015].

Jackson, Giorgio. El país que soñamos. Santiago de Chile: Debate, 2013.

Jasper, James. "Las emociones y los movimientos sociales: veinte años de teoría e investigación", Revista Latinoamericana de Estudios sobre Cuerpos, Emociones y Sociedad, 10/4 (2012): 48-68.

Kvale, Steinar. Den kvalitativa forskningsintervjun. Lund: Studentlitteratur, 2007.

Le Monde diplomatique. "Llamamiento de Democracia para Chile: Plebiscito Ahora!". Disponible en http://www.lemondediplomatique.cl/Llamamientode-Democracia-para.html, 2011. [Consultado el 07 de septiembre de 2013].

Marti i Puig, Salvador (s/f) "Los movimientos sociales". Disponible en http:// campus.usal.es/ -dpublico/areacp/materiales/Losmovimientossociales.pdf. [Consultado el 15 de mayo de 2015].

Mayol, Alberto. Derrumbe del modelo. La crisis de la economía de mercado en el Chile contemporáneo. Santiago de Chile: LOM, 2012.

McCarthy y Zald (1977) "Resource mobilization and social movements: A partial theory", American Journal of Sociology, 82/6 (1977): 212-1241. 
McCarthy, John \& Mayer N. Zald. “The Enduring Vitality of the Resource Mobilization Theory of Social Movements." In Turner, Jonathan H. (Ed.), Handbook of sociological theory (2001): 533-565.

Obsershall, Anthony. Social conflict and social movements. New York: Prentice-Hall, 1972.

Olson, Mancur. "La lógica de la acción colectiva". En Diez textos básicos de Ciencia Politica. Madrid: Editorial Ariel, (2001): 203-220.

Pérez, Simón. "El Mercurio y su "ethos totalitario", Perspectivas de la comunicación, 6/2 (2013): 65-78.

Picazo, María Inés. "Circulen, no hay nada que negociar". Democracia y Participación Universitaria. Santiago de Chile: Ediciones Universidad de Chile, 2013.

Puricelli, Sonia. "La teoría de movilización de recursos desnuda en América Latina", Revista Theomai, 12 (2005). Disponible en: http://www.redalyc. org/articulo.oa?id=12401202. [Consultado el 27 de mayo de 2015].

Somuano, María Fernanda. "Movimientos sociales y partidos políticos en América Latina: una relación cambiante y compleja”, Revista Politica y Cultura, 27 (2007): 31-53.

Svampa, Maristella. Protesta, movimientos sociales y dimensiones de la acción colectiva en América Latina. Disponible en http://maristellasvampa.net/ archivos/ensayo57.pdf, 2009. [Consultado el 15 de abril de 2015].

Tarrow, Sidney. El poder en movimiento. Los movimientos sociales, la acción colectiva y la politica. Madrid: Alianza Editorial, 1994.

Tilly, Charles. From Mobilization To Revolution. Michigan: University of Michigan, 1977.

Tilly, Charles. Los movimientos sociales de 1768 hasta el 2008. Desde sus origenes hasta el nacimiento del Facebook. Barcelona: Editorial Crítica, 2009.

Torres Nabel, Luis. “¿Quién programa las redes sociales en Internet? El caso de Twitter en el movimiento \#Yosoy132 México", Revista Internacional de Sociología, 73/2, 2015. 
UNICEF. La Voz del Movimiento Estudiantil 2011. Educación Pública, Gratuita y de Calidad. Santiago de Chile: Edición Miguel Lafferte y Carolina Silva, 2014.

Valderrama, Lorena. “Jóvenes, Ciudadanía y Tecnologías de Información y Comunicación. El movimiento estudiantil chileno", Revista Latinoamericana de Ciencias Sociales, Niñez y Juventud, 11/11 (2013): 123-135.

Valenzuela, Katia. "La primavera de Chile y sus matices: análisis del Movimiento Estudiantil y sus tensiones entre la política "desde abajo" y la apuesta institucional”. Avance de investigación en curso GT20 - Sociedad Civil: Protestas y Movimientos Sociales, 2012.

Zibechi, Raúl. "Movimientos sociales: nuevos escenarios y desafíos inéditos", Publicaciones OSAL, Observatorio Social de América Latina, Año VII/ 21 (2006): 221-230.

Zibechi, Raúl. Territories in Resistance: A Cartography of Latin American Social Movements. Edinburgh: AK Press Distribution, 2012. 